\title{
Asymmetry of W7-X magnet system introduced by torus assembly
}

\author{
Joris Fellinger, Konstantin Egorov, Johannes Peter Kallmeyer, Victor Bykov, Felix Schauer \\ Max-Planck-Institut Für Plasmaphysik, Teilinstitut Greifswald, Greifswald, Germany
}

\begin{abstract}
The magnet system of the stellerator Wendelstein 7-X (W7-X) consists of 5 modules of 14 superconducting coils with complex 3D shape each. After manufacturing the coils and assembly of the modules on temporary stands, the position of each module on the machine base was successfully optimized to minimize the electromagnetic (EM) field asymmetry. This asymmetry originates from inevitable geometric deviations of the coils from the target shape due to manufacturing and assembly tolerances.

However, new deviations were introduced after module optimization due to bolting the modules of the magnet system together to a torus, removing temporary supports and further loading of the machine base with weight of additional components.

In this paper, the geometrical deviations along the centre line of the coil currents are assessed through detailed step-by-step non-linear finite element (FE) simulation of the assembly procedure of the complete torus. The model is evaluated against measured displacements and reaction forces monitored during consequent assembly steps. The results are being used to quantify the obtained field asymmetry and countermeasures to minimize it.
\end{abstract}

Keywords: Wendelstein 7-X, magnet system, EM field error, torus assembly, FE modeling

\section{Introduction}

The magnet system of the stellerator Wendelstein 7-X (W7-X) consists of 5 modules. Each module consists of two flip symmetric half modules. Each half module is build up from $1 / 10^{\text {th }}$ of the central support ring (CSR) on which 5 non planar (NPC) and 2 planar (PC) superconducting coils of different types are bolted, see fig. 1. Welded, bolted and sliding inter-coil supports between the coils enhance the stiffness of the magnet system.

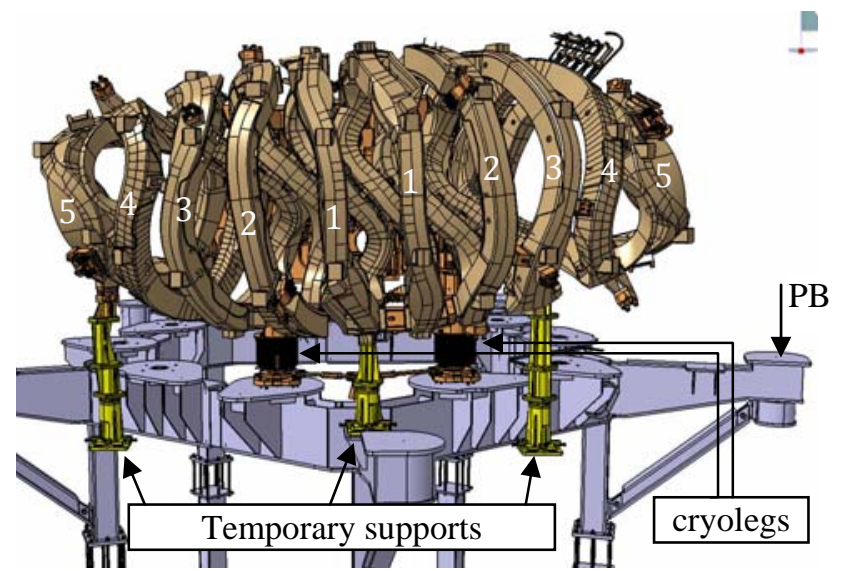

Fig. 1. CAD view of 1 module of magnet system (PCs not shown) on the machine base with temporary supports (yellow).

One of the major goals of W7-X is to attain best possible confinement of the plasma.

Manufacturing and assembly tolerances cause inevitable geometric deviations of the coil winding packs from the target shapes. Such deviations result in asymmetric field perturbations which disturb the confinement of the plasma. Hence they limit the performance of W7-X [1], even though they can partially be compensated by the trim coil outside the cryostat and control coils inside the plasma vessel [2]. Notably, asymmetry causes an uneven distribution of thermal loads on the divertors resulting in undesired high peak loads.

After manufacturing the coils and assembling the modules on temporary mounting stands, the position of each module on the machine base was successfully optimized to counterbalance field asymmetry caused by measured deviations from the target shape of the winding pack within the modules [1]. However, several assembly steps after module optimization inevitably introduced new asymmetry which needs to be quantified.

The objective of the presented work is to assess the asymmetry that developed during the assembly steps of the magnet system after the modules were placed in the optimized position.

In the next section the assembly of the torus from complete modules with the corresponding measurements of reaction forces and displacements are discussed. In section 3, the FE modeling is given and in section 4 the results are evaluated. Finally in section 5, the conclusions and recommendations are presented.

\section{Torus assembly}

\subsection{Assembly steps}

The starting point of the assessment is the optimization of the position of the first module on the machine base. Each module is placed in optimal position on the machine base, resting on two permanent cryolegs and three additional temporary supports under the NPCs, see fig. 1. Temporary supports are needed to keep the module stable as long as the modules are not interconnected. The middle temporary support (under NPC1) is standing on the machine base, while the other two (under NPC4 and NPC5) are supported on the first floor of the torus hall. 
The assembly continues with the positioning and optimization of the other 4 modules, the interconnection of the modules and the removal of temporary supports. The temporary supports under NPC4 and NPC5 were removed in one step (per module), the temporary supports under NPC1 were removed it two steps: first the force was reduced module per module until $1.5 \mathrm{~mm}$ sag was measured, then the remaining force was removed. In the final stage, all modules are interconnected and all temporary supports are removed, i.e. all weight is supported by the cryolegs on the machine base. Asymmetry is caused by:

- Removal of the temporary supports. Each module is supported on two permanent cryolegs under the CSR and three temporary supports under the NPCs, making each module statically undetermined, i.e. the distribution of the weight on the supports is not determined by equilibrium alone but also by the initial vertical position of the supports. Measured reaction forces on one type of temporary support differ up to $100 \mathrm{kN}$ between modules. Removal of the supports thus leads to different displacements between the modules.

- The first module (\#5) was optimized on the machine base before the machine base was loaded with the weight of the other modules and the major part of the cryostat. The last module (\#3) was optimized after most weight was applied to the machine base. Due to the deformation of the machine base the first module is further displaced from its optimized position than the last module.

- Some temporary supports were removed before the CSR was closed, and some modules were interconnected before all other modules were positioned. So the displacement field of some asymmetric stage was frozen in when the shims for the bolted CSR connections were machined to match the measured gap between the CSR flanges.

\subsection{Measurements}

The positioning and optimization of each module of the machine base was accompanied by measurements of the reaction forces in the cryolegs and in temporary supports. For that purpose, the temporary supports and cryolegs were instrumented with 3 and 6 strain gauges (SGs) respectively. The SGs were read out before and after each assembly step. They were calibrated using the measured forces in the hydraulic cylinder beneath each temporary support and cryoleg. The cylinders could only be read out when they were activated, i.e. during positioning and optimization of the corresponding module and during removal of the corresponding temporary support. Unfortunately, the hydraulic forces were not very accurate: The sum of the measured hydraulic forces per module varied between 860-970 kN although the mass of the modules should be very similar. The forces in the hydraulic cylinders were also not in rotational equilibrium around the centre of gravity of the modules (centre according to FE model of chapter 3). Moreover, some supports were not instrumented, some SGs were broken and the calibration of the SGs with the hydraulic forces showed high scatter. This was attributed to unintended bending moments in the supports. Notably, the temporary supports and cryolegs were not supported with perfect hinges and the instrumented cross sections were not perfectly symmetric, thus bending moments were not fully cancelled out among the SGs.

Besides, displacements of marked reference points on the magnet system were measured with laser trackers during removal of temporary supports. Relative displacements could be measured within $0.3 \mathrm{~mm}$ accuracy. These measurements have been used to validate the FE model.

\section{FE modeling}

Starting point was the existing $72^{\circ}$ model of one module in Abaqus v6.11 [3]. This model was multiplied 5 times to obtain a full $360^{\circ}$ model. The machine base including the torus hall construction and temporary supports were added, see fig. 2.

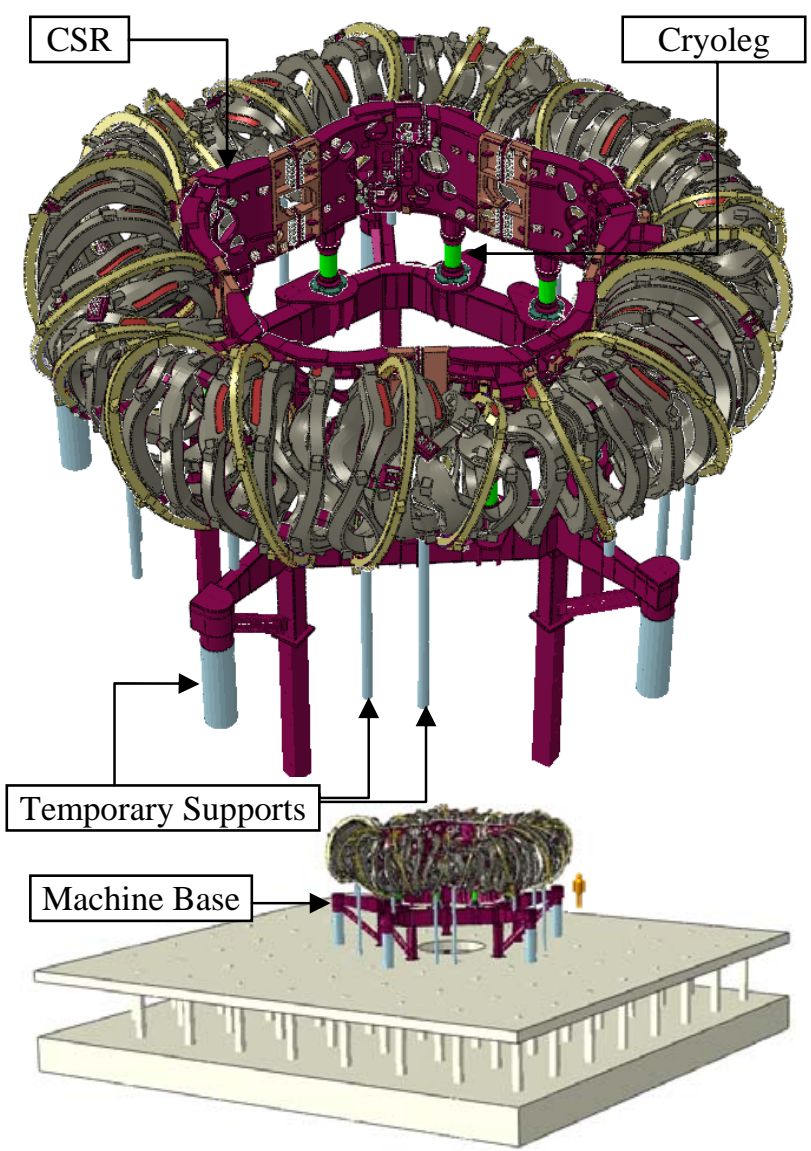

Fig. 2. $360^{\circ} \mathrm{FE}$ model of magnet system and machine base only (top) and complete model (bottom).

The temporary supports were modeled with prestressed beam elements for two different reasons: Under NPC4 and NPC5 to be able to load the support with the measured force during the first step of module optimization, and under NPC1 to be able to unload with defined displacement. Only the initial force under NPC4 and NPC5 was prescribed, so the three forces under NPC1 and in the cryolegs follow directly from vertical and rotational equilibrium. 
To reflect the uncertainties in the force measurements, FE simulations were carried out once with the measured initial forces under NPC4 and NPC5 that are different for each module and once with equal "target" forces in each module calculated as the average measured forces but slightly corrected to fulfill rotational equilibrium, see table 1 .

Since the FE model does not include non-structural masses such as cooling pipes and busbars, gravitation was adapted per module to match the FE mass with the measured module weight.

Table 1: Measured, mean and target forces in temporary supports and cryolegs (CL) and the resulting moments

\begin{tabular}{lccccccc}
\hline & \multicolumn{7}{c}{ Measured reaction forces [kN] } \\
\cline { 2 - 7 } Total weight & Mo1 & Mo2 & Mo3 & Mo4 & Mo5 & mean & \\
\cline { 2 - 7 } & 972 & 914 & 912 & 906 & 860 & 912 & 912 \\
\hline NPC5 & 166 & 149 & 154 & 172 & 71 & 153 & 155 \\
CL1 & 62 & 83 & 72 & 79 & 164 & 92 & 95 \\
NPC1 & 465 & 402 & 368 & 355 & 374 & 381 & 364 \\
CL2 & 58 & 95 & 90 & 52 & 62 & 71 & 74 \\
NPC4 & 221 & 185 & 228 & 248 & 189 & 214 & 224 \\
\hline Mx & 26 & 24 & -62 & 13 & -109 & 14 & 0 \\
My & -145 & -21 & -8 & -35 & 24 & -22 & 0 \\
\hline
\end{tabular}

During assembly the machine base is not only loaded with the magnet system but also with increasing weight of the assembled cryostat system, consisting of the outer vessel (OV), the plasma vessel (PV) with plasma facing components (PFC) and ports in between PV and OV. Since the assembly of the cryostat follows more or less the modular sequence of the magnet system, it was decided to simply add some cryostat weight (98 kN) during the step of module optimization (lower shell of the OV plus the PV) and the rest $(292 \mathrm{kN})$ in the step directly after module optimization. The distribution of these loads on the MB was determined with a separate FE model of the cryostat [4], see table 2 .

Table 2: Additional cryostat weight on the machine base.

\begin{tabular}{lcccc}
\hline assembly stage & \multicolumn{4}{c}{ load [kN] } \\
\cline { 2 - 5 } & total & CL1 & CL2 & PB $^{*}$ \\
\hline during module optimization & 98 & 25 & 14 & 59 \\
after module optimization & 292 & 73 & 42 & 177 \\
\hline
\end{tabular}

*) Load on protruding beam of machine base, see fig. 1.

All temporary supports were removed in a smooth way by weakening their Young's modulus via artificial temperature dependence.

Bolting the modules together at the CSR and intercoil support structures was modeled using initial suppression and later activation of one layer of solid elements at the interface. In doing so, these elements are stress free at the moment of activation despite the deformed shape of modules at both sides of the interface.

The final simulation steps are given in table 3 . Element activation to connect modules is done in step 511 according to assembly.

To speed up the calculation, all bolted contacts were fixed and all sliding contacts between sliding inter-coil supports were removed because calculation showed that the bolted connections do not slide and the sliding contacts remain open without electromagnetic loads. The contact between the winding pack and the coil case was also fixed which dramatically reduced the calculation time to 0.2-0.5 days per step on 2 Intel X5260 CPUs depending on the convergence speed. To evaluate the stiffness contribution of this simplification, the calculation was repeated with 100 fold lower stiffness of the winding pack. So, in total three simulations were done: With measured initial force and normal winding pack stiffness (case 1a), the same with reduced winding pack stiffness (1b) and with the same target initial forces in the temporary supports for all modules (2). To check the effect of the sequential removal of temporary supports under NPC1, step 13-22 of case 2 were repeated in one step removing all temporary supports under NPC1 simultaneously (2'), see table 4.

Table 3: Overview of simulation steps.

\begin{tabular}{cl}
\hline 1 & Preloading all bolts in all modules \\
\hline $2-6$ & Adding module weight + cryostat weight $(5 \mathrm{x})$ \\
7 & Adding remaining cryostat weight \\
\hline $8-12$ & Removing temp. supports at module interfaces $(5 \mathrm{x})$ \\
$13-17$ & $1.5 \mathrm{~mm}$ sag under NPC1 for each module $(5 \mathrm{x})$ \\
$18-22$ & Removal of remaining load under NPC1 $(5 \mathrm{x})$ \\
\hline
\end{tabular}

Table 4: Overview of simulation cases

\begin{tabular}{cl} 
1a & Measured initial forces, normal winding pack stiffness \\
1b & Measured initial forces, weak winding pack stiffness \\
\hline 2 & Equal initial forces, normal winding pack stiffness \\
2' & Removal of load under NPC1 in one step
\end{tabular}

\section{Results}

First, the calculated displacements during removal of the temporary supports at the module interfaces (step 812) are compared with the measurements, see fig. 3. Vertical displacements were measured at NPC4 of the initial (I) side of the each flip symmetric module and at NPC5 of the reflected (R) side of the adjacent module at the other side of the module interface. It shows a systematic overestimation of the displacements by a factor 1.3-2.2.

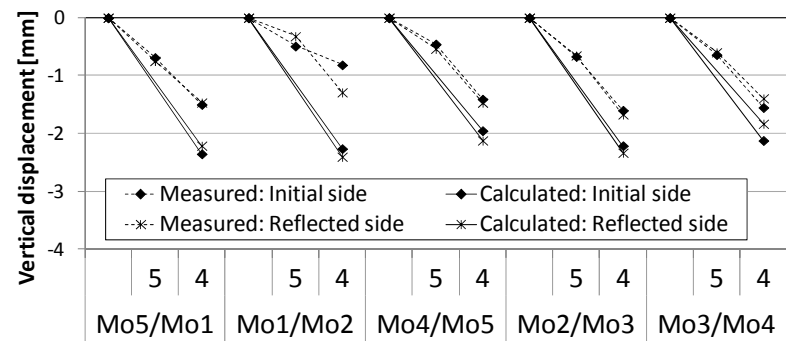

Fig. 3. Calculated (case 2) vs. measured displacements during removal of temporary supports under coil 5 and coil 4 near the module interface.

Also the incremental displacements during removal of the temporary supports under NPC1 were compared, see fig. 4. The measured displacements are plotted against the predicted forces base on case 1a. In this case, the displacements are only overestimated by a factor 11.3. Notably, with the removal of the temporary supports at the module interface, the removed load is added to the 
machine base, whereas with the removal of the temporary supports under NPC1, there is only a redistribution of loads within the machine base. It suggests that the FE model of the machine base and torus hall is generally too flexible.

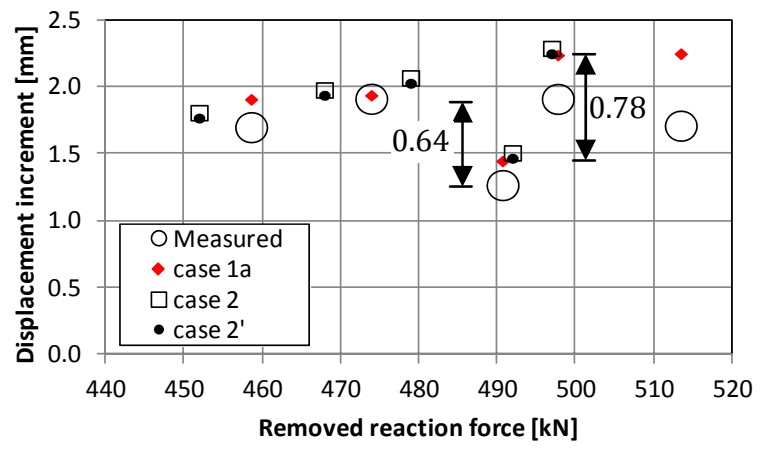

Fig. 4. Calculated vs. measured displacements during removal of temporary supports under NPC1.

Nevertheless, the predicted asymmetry during removal of temporary supports under NPC1of maximum $0.78 \mathrm{~mm}$ difference between modules matches the measured $0.64 \mathrm{~mm}$ satisfactory. The module per module removal of the temporary supports under NPC1 leads to almost the same incremental displacement as the simultaneous removal of all five temporary supports.

Before torus assembly, the asymmetry had already been assessed with an independent $360^{\circ} \mathrm{FE}$ model in Ansys, which was created by five time multiplication of the default Ansys model of one module [3]. The model does not include the machine base. As a result, the model is stiffer than the Abaqus model. It simulated the originally planned assembly process which deviates slightly from the final procedure. It assumed equal initial loads in the temporary supports and a simultaneous removal of the temporary supports under NPC1. According to this model, the asymmetry due to removal of temporary supports under NPC1 is limited to $0.19 \mathrm{~mm}$. The difference with Abaqus and with the measurements is partly caused by the deviating assembly steps leading to smaller differences between modules concerning the loads in the temporary supports under $\operatorname{NPC1}(\Delta \mathrm{F}=30 \mathrm{kN}$ in Ansys vs $\Delta \mathrm{F}=54$ in Abaqus $)$ and partially due to the rigidly modeled support under the magnet system.

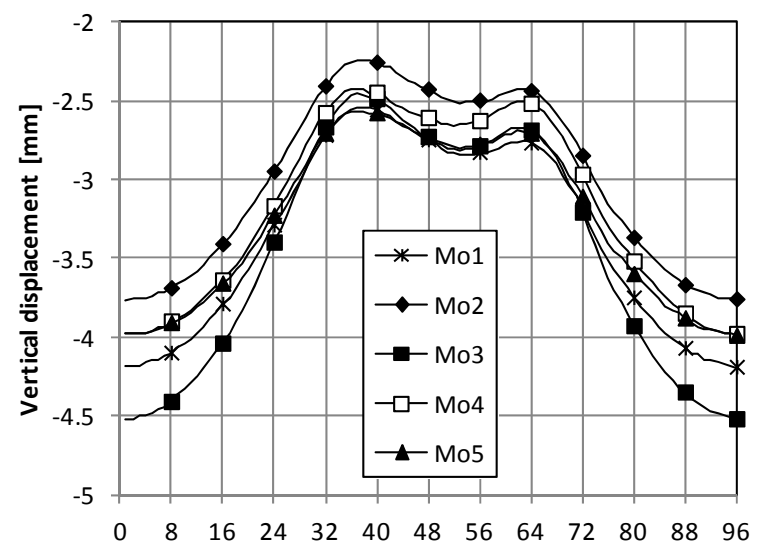

Fig. 5. Incremental vertical displacements along the winding pack axis of NPC1 during torus assembly.
Next step is the assessment of the asymmetry of the winding pack. For that purpose the displacement vector was extracted from the results along the centre line of the winding pack of each coil. As an example, the incremental displacement from module optimization to the final assembled stage is plotted in fig. 5 along the winding pack axis of NPC1 subdivided in 96 segments. In table 5 , the asymmetry is given in terms of maximum incremental displacement differences between modules. These calculated values will be used in the future to assess the effect of the asymmetry on the EM field error since the winding pack is not accessible anymore for a direct measurement.

Table 5. Maximum differences between modules of incremental winding pack displacements

\begin{tabular}{|c|c|c|c|c|c|c|c|c|c|}
\hline \multirow[b]{2}{*}{ direction } & \multirow[b]{2}{*}{ case } & \multicolumn{5}{|c|}{ NPC } & \multicolumn{2}{|c|}{ PC } & \multirow[b]{2}{*}{ Max } \\
\hline & & 1 & 2 & 3 & 4 & 5 & $\mathrm{~A}$ & $\mathrm{~B}$ & \\
\hline \multirow{3}{*}{ radial } & $1 \mathrm{a}$ & 1.8 & 1.8 & 1.7 & 1.6 & 1.5 & 2.0 & 1.8 & \multirow{3}{*}{2.0} \\
\hline & $1 b$ & 1.4 & 1.4 & 1.4 & 1.4 & 1.4 & 1.5 & 1.4 & \\
\hline & 2 & 1.8 & 1.8 & 1.7 & 1.6 & 1.4 & 1.9 & 1.6 & \\
\hline \multirow{3}{*}{ toroidal } & $1 \mathrm{a}$ & 0.9 & 0.9 & 0.9 & 0.9 & 1.1 & 0.9 & 1.1 & \multirow{3}{*}{1.3} \\
\hline & $1 b$ & 0.9 & 0.9 & 1.1 & 1.3 & 1.3 & 1.0 & 1.3 & \\
\hline & 2 & 0.7 & 0.7 & 0.8 & 0.9 & 1.0 & 0.7 & 1.0 & \\
\hline \multirow{3}{*}{ vertical } & $1 \mathrm{a}$ & 0.9 & 1.0 & 1.1 & 1.1 & 1.3 & 1.0 & 1.3 & \multirow{3}{*}{1.3} \\
\hline & $1 \mathrm{~b}$ & 0.8 & 0.9 & 1.0 & 1.0 & 1.2 & 0.9 & 1.3 & \\
\hline & 2 & 0.8 & 0.8 & 0.7 & 0.6 & 0.7 & 0.9 & 0.7 & \\
\hline
\end{tabular}

\section{Conclusions}

Concerning the asymmetric position of the winding pack which deteriorates the EM confinement of the plasma, the assembly of all five modules of the magnet system of W7-X towards a closed torus was successfully simulated in 22 steps with a $360^{\circ} \mathrm{FE}$ model of the magnet system including the machine base and temporary supports.

The incremental displacements differ between modules up to 2.0, 1.3 and $1.3 \mathrm{~mm}$ in the radial, toroidal and vertical direction, respectively. Since the model tends to overestimate the displacements compared to measurements, these values can be considered as an upper bound.

The effect of the winding pack stiffness is small. As expected, slightly smaller differences between modules are found if one assumes the same initial reaction forces in each module after module optimization. The magnet system responds nearly linear as is shown by the negligible differences between the sequential and simultaneous removals of all temporary supports under NPC1.

The asymmetry introduced by the torus assembly is of the same order of magnitude as the expected asymmetry of $1.8 \mathrm{~mm}$ differences between modules due to expected variation of uncertain parameters like Young's modulus, initial gaps between sliding supports, bolt pre-stress and friction coefficients of bolted contacts, see [5].

The obtained differences will be used as input of the coil positions to calculate the EM field error. 


\section{References}

[1] T. Andreeva et al., Evaluation of W7-X magnetic field perturbations during optimized module positioning, Proc. 39th EPS conf. Stockholm, Sweden (2012)

[2] J. Kißlinger et al., Magnetic field accuracy and trim coils in W7-X, Fus. Sc. \& Tech. 50, 3 (2006) pp 382-386

[3] V. Bykov et al., Structural analysis of W7-X: Overview, Fus. Eng. \& Des. 84, 2-6 (2009), pp. 215-219

[4] A. Tereshchenko et al., FE simulation of the Wendelstein 7-X cryostat system, Fus. Eng. \& Des. 84, 7-11 (2009), pp. 1833-1837

[5] T. Andreeva et al. Influence of assembly and operation asymmetries on Wendelstein 7-X magnetic field perturbations, proc. $40^{\text {th }}$ EPS conf., Espoo, Finland 